\title{
Disruption of Trichoderma reesei cre2, encoding an ubiquitin C-terminal hydrolase, results in increased cellulase activity
}

\author{
Jai A Denton and Joan M Kelly
}

\begin{abstract}
Background: The filamentous fungus Trichoderma reesei (Hypocrea jecorina) is an important source of cellulases for use in the textile and alternative fuel industries. To fully understand the regulation of cellulase production in $T$. reesei, the role of a gene known to be involved in carbon regulation in Aspergillus nidulans, but unstudied in $T$. reesei, was investigated.

Results: The T. reesei orthologue of the A. nidulans creB gene, designated cre2, was identified and shown to be functional through heterologous complementation of a creB mutation in A. nidulans. A T. reesei strain was constructed using gene disruption techniques that contained a disrupted cre2 gene. This strain, JKTR2-6, exhibited phenotypes similar to the $A$. nidulans creB mutant strain both in carbon catabolite repressing, and in carbon catabolite derepressing conditions. Importantly, the disruption also led to elevated cellulase levels.

Conclusions: These results demonstrate that cre2 is involved in cellulase expression. Since the disruption of cre 2 increases the amount of cellulase activity, without severe morphological affects, targeting creB orthologues for disruption in other industrially useful filamentous fungi, such as Aspergillus oryzae, Trichoderma harzianum or Aspergillus niger may also lead to elevated hydrolytic enzyme activity in these species.
\end{abstract}

\section{Background}

Environmental sustainability and fossil fuel supply concerns have caused increased focus and research activity in the area of alternative fuel production. The filamentous fungus Trichoderma reesei (Hypocrea jecorina) has been used for cellulase production over many decades, and the release of the complete genome sequence facilitates studies that will extend our understanding of how cellulases are regulated in this organism. This understanding may present further opportunities for targeted gene manipulation to increase cellulolytic enzyme production. In the presence of glucose and other easily metabolised carbon sources, genes encoding cellulase enzymes are not highly expressed, even when inducer is present, due to carbon catabolite repression [Reviewed [1,2]]. An industrially important cellulolytic strain of $T$. reesei, Rut-C30, was selected for improved cellulase production following multiple rounds of mutagenesis and

* Correspondence: joan.kelly@adelaide.edu.au

School of Molecular and Biomedical Science, University of Adelaide, Adelaide, 5005, Australia selection. The Rut-C30 genome contains a mutation in the gene encoding a transcriptional repressor involved in carbon catabolite repression, cre1 [3]. Many other mutations have also been identified in Rut-C30 [4-6], and those analysed after identification by array comparative genomic hybridization, including an $85 \mathrm{~kb}$ deletion containing 29 genes, do not affect cellulase production [6]. These studies show that mutagenesis and selection lead to many off target genetic changes, and some of these may be undesired. A complete understanding of the complex regulation of cellulose metabolism may open up the opportunity for precise targeted genetic manipulation of T. reesei strains for increased cellulase production.

Genetic analysis in the model filamentous fungus, Aspergillus nidulans, has provided a framework for the study of the mechanism of carbon repression in $T$. reesei. In A. nidulans there are three genes in which mutations have been identified resulting in partial deregulation of carbon repression: $c r e A$, the $A$. nidulans orthologue of cre1, encoding a zinc finger DNA binding transcriptional repressor $[7,8]$; cre $B$, encoding a 
ubiquitin C-terminal hydrolase [9]; and $\mathrm{cre} C$, encoding a WD40 repeat protein shown to be present in a complex with CreB [10]. Orthologues of $c r e A$ have been identified and studied in numerous fungi including $A$. niger [11], Humicola grisea [12], Cochliobolus carbonum [13], Gibberella fujikuroi [14] and Botrytis cinerea [14], however $c r e B$ and $c r e C$ orthologues remain largely unstudied in other fungi. Mutations in the $\mathrm{creB}$ and $\mathrm{cre} C$ genes lead to partial deregulation of carbon repression of some genes, and affect the expression of other genes, but they do not cause the severe morphological impairment caused by creA mutations [15]. In A. nidulans there is evidence that CreA is a direct target of $\mathrm{CreB}$ suggesting that $\mathrm{CreB}$ functions in carbon repression via CreA [16]. Disruption of carbon source mediated repression without severe morphological impairment could potentially lead to the development of industrially useful fungal strains, and as such $c r e B$ represents a candidate for targeted disruption.

The deletion of cre1 within the progenitor strain of $T$. reesei, QM 6a, has recently been characterised [17]. As with $A$. nidulans strains containing creA mutations, the deletion of cre1 in this non mutated background resulted in higher total secreted protein and enzymatic activity of endoglucanases and xylanases, as well as morphological impairment. Cellulase regulation in $T$. reesei has also been shown to involve transcriptional regulators including the transcriptional repressor Ace1 [18,19], and the transcriptional activators and Ace2 [20] and Xyr1 $[21,22]$. It has recently been shown that xyr 1 transcription is repressed by $\mathrm{Cre} 1$, and it was proposed that Ace1 might also play a role in $x y r 1$ repression [23]. Deletion of xyr 1 in $T$. reesei resulted in loss of transcription of the major cellulase encoding genes, cbh1, cbh2 and egl1 [22], and furthermore there was no secreted cellulase or xylanase activity detected in the xyr 1 deletion strain after 72 hours growth in inducing conditions [22]. Xyr1 has also been implicated in the expression of Egl3 [24] and the induction of the lactose metabolism pathway by lactose [25].

We find no sequence similar to ace 2 in the $A$. nidulans genome, highlighting the differences between regulation of the cellulases in the two filamentous fungi. In this study we have shown bioinformatically that the $T$. reese $i$ genome contains orthologues of the $A$. nidulans cre $B$ and cre $C$ genes. There have been no reports of mutations in cre $B$ and $c r e C$ orthologues in $T$. reese $i$, and it was possible that mutations in the $T$. reese $i$ orthologues of $c r e B$ or cre $C$ may not result in the equivalent phenotypes seen in A. nidulans, as regulation of cellulase encoding genes in $T$. reesei could have been independent of the $\mathrm{CreB} / \mathrm{CreC}$ complex. We have cloned the cre 2 gene and shown that it is a functional orthologue of the $A$. nidulans $c r e B$ gene. A $T$. reesei strain containing a disruption within the $c r e 2$ encoding region was generated and the phenotypic effects analysed.

\section{Results}

The $T$. reesei Genome Contains Homologues of $c r e B$ and creC

The $T$. reesei genome was examined to identify potential orthologues of the $A$. nidulans creB and cre $C$ genes. Using protein sequences for CreB (AAL04454) and CreC (AAF63188) from the National Centre for Biotechnology Information website http://www.ncbi.nlm.nih.gov, TBLASTN analyses were performed against the $T$. reese $i$ translated nucleotide database http://genome.jgi-psf.org/ Trire2/Trire2.home.html. Both the identified genomic regions and deduced protein sequences of the $T$. reesei $\mathrm{CreB}$ and $\mathrm{CreC}$ orthologues, designated $\mathrm{Cre} 2$ (Protein ID 122405) and Cre3 (Protein ID 64608) were aligned to the A. nidulans orthologues using the Geneious software package (Biomatters Ltd, USA). The Cre 2 amino acid sequence showed $43 \%$ identity to $A$. nidulans $\mathrm{CreB}$ (Figure 1), while the Cre3 sequence showed $48 \%$ identity to $A$. nidulans $\mathrm{CreC}$.

\section{Cre2 is a Functional Orthologue of CreB}

An $A$. nidulans strain that contained the riboB2 and creB1937 mutations was transformed with pPL3 containing ribo $B^{+}$, with pTRcre 2 , containing $T$. reesei cre 2 also present in the transformation mix. The creB1937 mutation leads to a pleiotropic phenotype, including sensitivity to allyl alcohol in the presence of glucose and poor utilisation of proline. Six riboflavin independent transformants were tested for co-transformation of pTRcre2 via complementation of these creB1937 phenotypes (Figure 2). Transformants T3 and T6 were morphologically similar to wildtype (Figure 2a), and also showed complementation of the phenotypes caused by creB1937 of sensitivity to allyl alcohol in the presence of glucose (Figure $2 \mathrm{~b}$ ), and poor proline utilisation. T1, T2, T4 and T5 showed varying degrees of partial complementation, possibly due to differences in expression levels due to copy number or position of integration affects, but these were not studied further as the aim was to show that Cre 2 had the same function as CreB, rather than a complete analysis of the transformants. Thus the $T$. reesei cre 2 gene is a functional orthologue of $c r e B$, and cre 2 can be expressed in $A$. nidulans from the $T$. reesei cre 2 promoter.

\section{Generation of a cre2 Disruption Strain}

A plasmid, pTRcre $2 \Delta$ amdS, was constructed to disrupt cre 2 in $T$. reesei, in which the A. nidulans amdS gene containing the amdSI9 promoter mutation was inserted into the cre2 ORF disrupting the gene within the ubiquitin C-terminal hydrolase domain. The position of the 


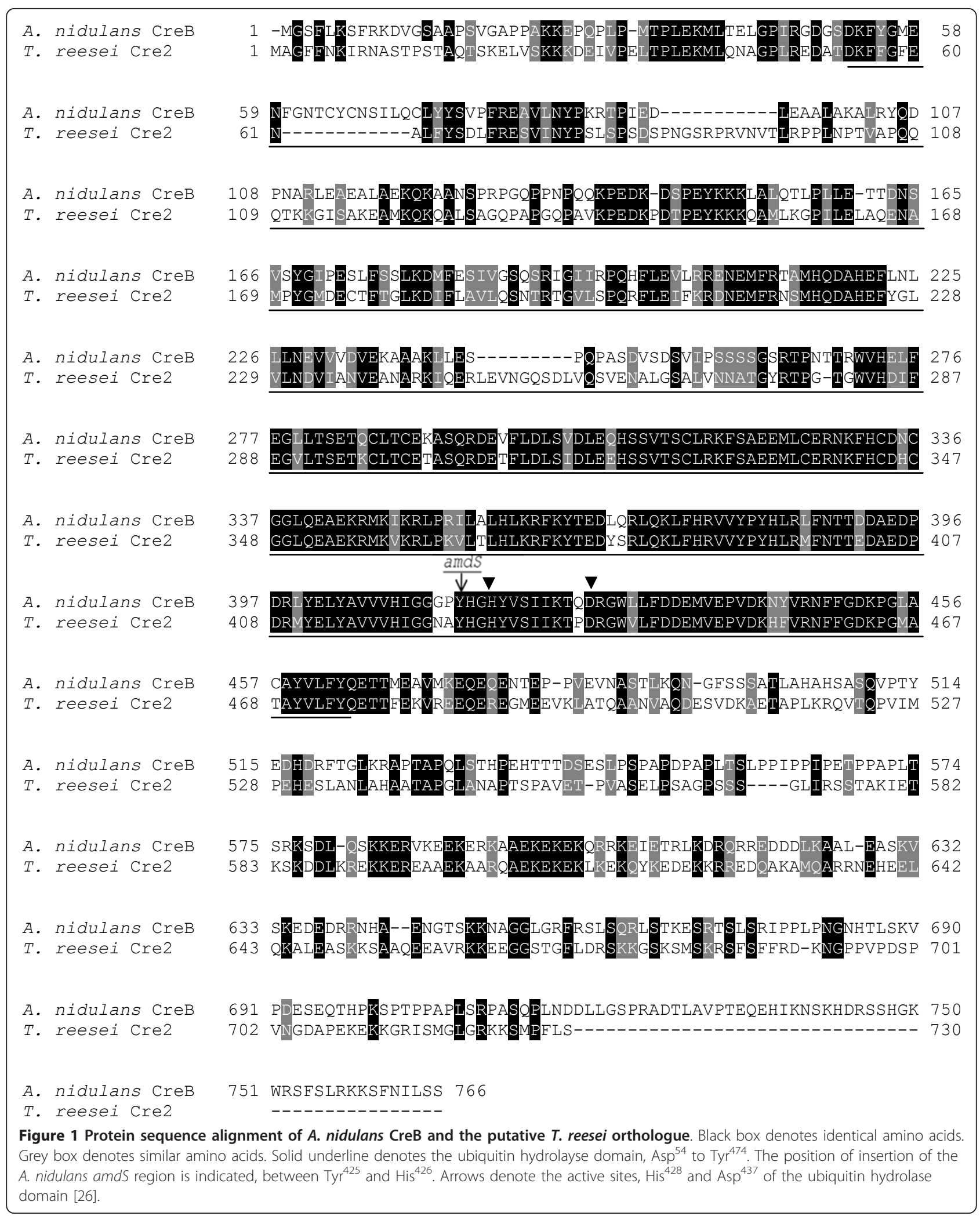



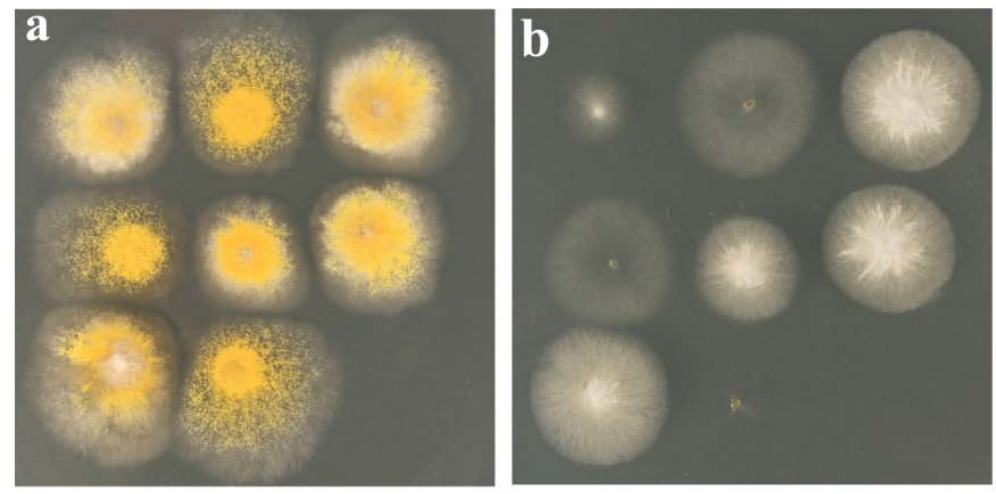

c

T1

T4

wt
T2

T3

T5

T6

Figure 2 Complementation of $\boldsymbol{A}$. nidulans creB1937 by $\boldsymbol{T}$. reesei cre2. The A. nidulans creB1937 mutant strain was co-transformed with pPL3 and pTRcre2 containing Trichoderma reesei cre2, and six riboflavine independent transformants, T1-T6, were tested for complementation of creB1937 phenotypes. Strains were incubated at $37^{\circ} \mathrm{C}$ on a) $1 \%$ glucose, $10 \mathrm{mM}$ ammonium tartrate; b) $1 \%$ glucose, $10 \mathrm{mM}$ ammonium tartrate, $10 \mathrm{mM}$ allyl alcohol; c) key to strains. Plates were supplemented with riboflavin and para amino benzoic acid.

insertion of the $A$. nidulans amdS encoding region is indicated in Figure 1, between $\mathrm{Tyr}^{425}$ and His ${ }^{426}$, which is prior to the essential active sites of the ubiquitin hydrolase domain, $\mathrm{His}^{428}$ and $\mathrm{Asp}^{437}$ [26], and thus this insertion will create a non-active allele. A strain containing a disruption of the endogenous cre2 gene, JKTR2-6, was generated by transformation of QM 6a with this plasmid. After purification, homologous recombination at the cre 2 locus, required for disruption of the ORF, was verified using Southern analysis.

\section{Meiotic Segregation of the Disruption Phenotype with the amdl9 Phenotype}

With the recent discovery of strains of opposite mating type [27], meiotic crossing can be performed in T. reesei. The disruption construct contains the amdI9 selection marker, and this can be followed in a genetic cross through scoring the strong growth on acrylamide. To provide evidence that the phenotypes observed were due to the disruption and not a fortuitous change elsewhere in the genome, JKTR2-6 was crossed to CBS999.97, a strain shown to undergo sexual reproduction with QM 6a [27]. The pleiotropic phenotype attributed to cre 2 disruption always segregated with the amdI9 phenotype in the progeny of a sexual cross. Of 38 progeny screened, 23 grew on acrylamide due to the amdI9 marker and all 23 also showed the pleiotropic phenotype. This segregation of the cre 2 disruption phenotype with the amdI9 marker shows that the pleiotropic phenotype seen in the disrupted strain segregates together and is not due to fortuitous changes elsewhere in the genome.

Phenotypic Effects of cre2 Disruption on mycelial growth JKTR2-6 showed similar mycelial growth but slightly reduced conidiation compared to QM 6a on solid minimal media with glucose as the carbon source, and considerably stronger mycelial growth and conidiation compared to the cre 1 deletion strain on this medium (Figure 3a). JKTR2-6 grew much better than QM 6a on solid medium containing maltose (Figure $3 \mathrm{~b}$ ), a phenotype linked to increased $\alpha$-glucosidase activity that has also been shown for $A$. nidulans strains containing $c r e B$ mutations [15], whereas the $T$. reesei cre1 deletion strain grew very poorly on this medium. QM 6a and JKTR2-6 grew similarly when cellobiose was the sole carbon source, suggesting limited effects on $\beta$-glucosidase activity (Figure 3c). JKTR2-6 showed poorer growth than QM 6a or the cre1 deletion strain on medium containing proline as the sole nitrogen source (Figure $3 \mathrm{~d}$ ), a phenotype also seen with $A$. nidulans creB mutant strains. However, unlike $c r e B$ mutations in $A$. nidulans which also grow poorly on lactose, JKTR2-6 had no discernable effect on lactose utilization in solid medium (data not shown) but effects were apparent in liquid conditions (see below). JKTR2-6 also had higher protease activity than QM 6 a on medium containing $5 \%$ ( $\mathrm{vol} / \mathrm{vol}$ ) liquid soy milk, as assessed by the size of the cleared zone around the colonies, but not when $1 \%$ glucose was also present in the medium.

Growth tests were also performed in $50 \mathrm{ml}$ shake flask culture. Dry biomass weights were determined for a range of carbon sources for each of the three strains (Figure 4; Additional File 1). JKTR2-6 had significantly $(\mathrm{P}<0.05)$ less biomass than QM 6a on glucose at 24 and 36 hours, but significantly $(\mathrm{P}<0.001)$ more than the cre 1 disruption. Solid media growth tests indicated greatly improved growth on maltose for JKTR2-6 and extremely poor growth for the cre 1 disruption, which was corroborated in the liquid culture experiments. JKTR2-6 had three times more biomass than QM 6a at 

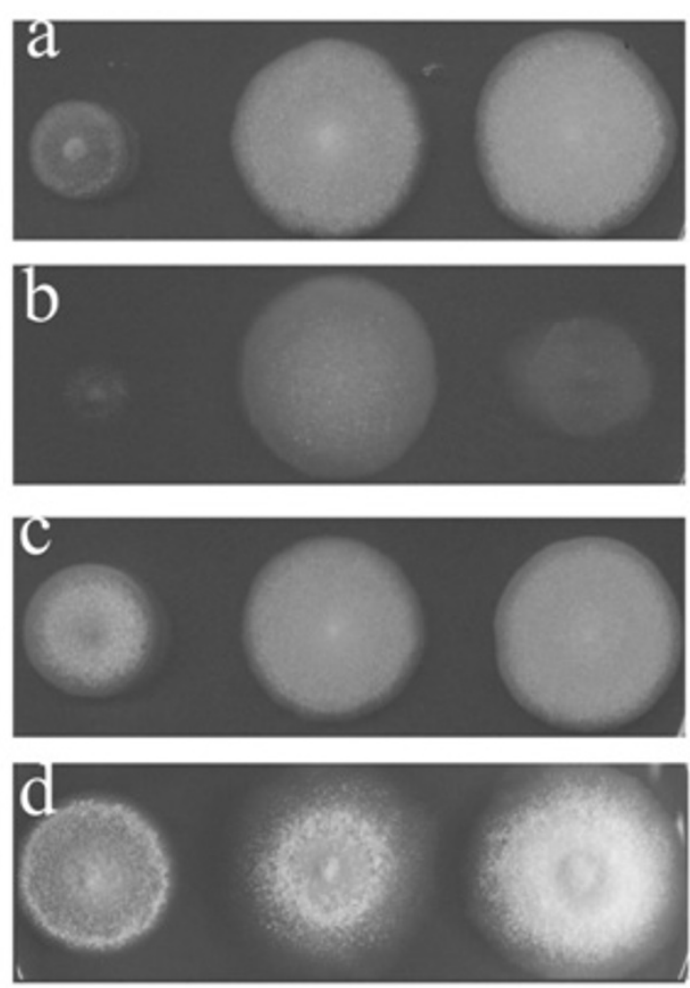

e

\section{cre1A JKTR6-2 QM6a}

Figure 3 Phenotype of $T$. reesei cre2 disruption strain on solid media. Strains were grown at $30^{\circ} \mathrm{C}$ on a) $1 \%$ glucose, $10 \mathrm{mM}$ ammonium tartrate; b) $1 \%$ maltose, $10 \mathrm{mM}$ ammonium tartrate; c) $1 \%$ cellobiose, $10 \mathrm{mM}$ ammonium tartrate; d) $1 \%$ glucose, $10 \mathrm{mM}$ proline; e) Key to strains. $0.01 \%$ triton- $X$ was added to all plates for photography, but plates without Triton-X showed essentially identical results.
24 hours post inoculation while the cre 1 disruption failed to grow. Analysis of liquid cultures showed that disrupting cre 2 reduced growth on lactose relative to QM 6a, a result that reflects the phenotype of creB mutations in $A$. nidulans but that was not detectable for the $T$. reesei mutation on solid medium. The disruption of cre 1 or cre 2 had no noticeable effect in medium with $2 \%$ glycerol as the sole carbon source, and both strains had similar amounts of biomass compared to QM 6 a. The growth of JKTR2-6 on 2\% sorbitol was very similar to QM 6a, while the cre1 disruption grew very poorly.

\section{Effects of cre2 Disruption on cellulase activity Sophorose as inducer}

In a preliminary experiment, total secreted cellulase activity was assayed in $5 \mathrm{ml}$ shake cultures in medium containing glucose as a repressing carbon source and sophorose as an inducer. When glucose was present, JKTR2-6 and the cre1 deletion strain showed high levels of secreted cellulase activity after 6,12 and 18 hours, whereas QM 6a had limited cellulase activity after 6 and 12 hours, but the activity had increased to levels similar to the disruption strains by 18 hours. Glucose levels were determined in QM 6a culture media using HPLC, and were $0.03 \%$ at 12 hours and $0 \%$ at 18 hours, and thus by 18 hours the cultures contained no source of repression. When sorbitol, a nonrepressing carbon source, replaced glucose, JKTR2-6 showed higher cellulase activity than either QM 6a or the cre1 deletion strain at all time points (Additional File 2). These experiments indicated that $\mathrm{cre} 2$ had an effect on cellulase expression, but the small culture volumes (due to sophorose cost), absence of accurate mycelia mass, and complications in dissecting effects on repression from those on uptake of sophorose [28] made interpretation

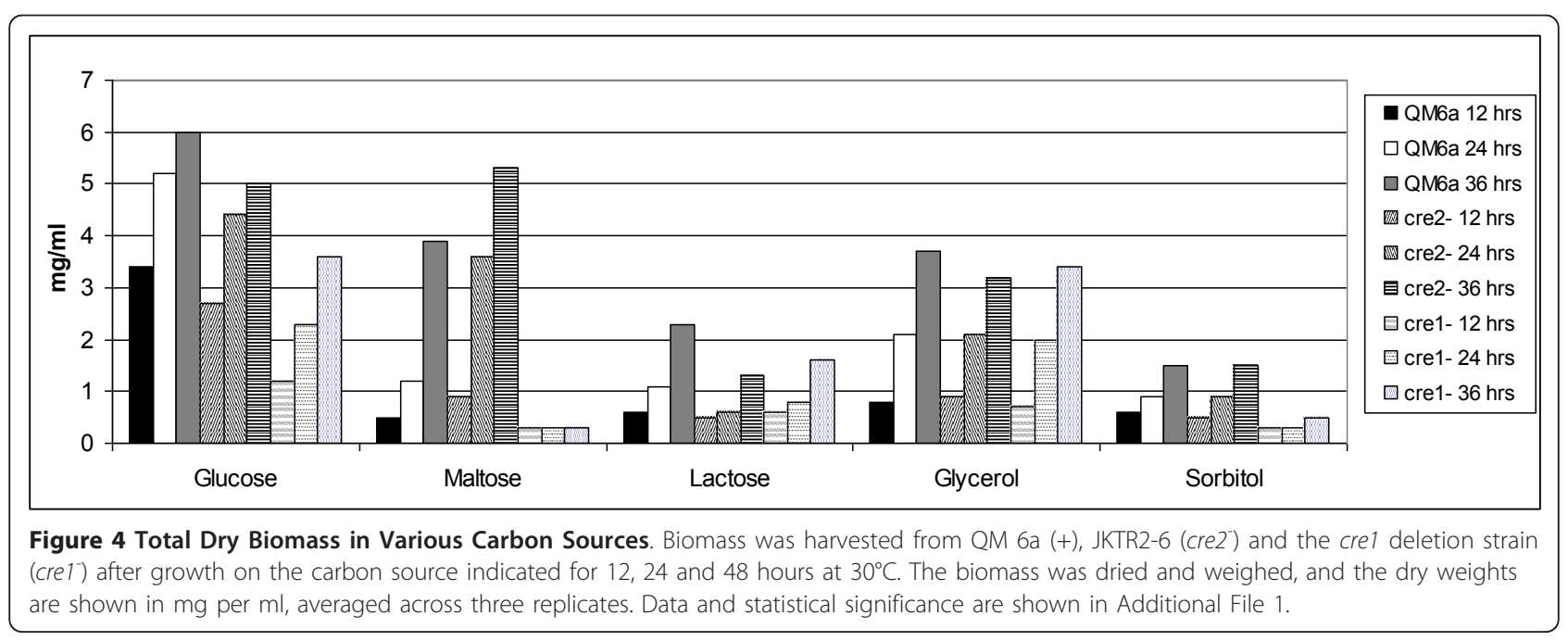


difficult. Based on these preliminary observations, we undertook further experiments in larger volumes using two inducers, lactose, the catabolism of which is initiated by extracellular hydrolysis, thus reducing the effects of inducer exclusion, and microcrystalline cellulose (Avicel).

\section{Lactose as inducer}

In experiments using lactose as the inducer, strains were grown in $50 \mathrm{ml}$ shake cultures, which allowed accurate measurement of mycelial dry weights to determine activity per gram dry weight. There was little cellulase activity detected in any of the strains tested in glucose (Figure 5a), sorbitol (Figure 5d) or glycerol (Figure 5e) media without added inducer. In medium containing both glucose and lactose as inducer, for QM 6a, cellulase activity was not detected as the cellulase encoding genes are subject to glucose repression. In the cre 1 deletion strain, cellulase activity was detected consistent with previous work indicating that at least one major exocellulase was not subject to carbon repression in the cre1 deletion strain (Figure 5b). In JKTR2-6 there was no detectable cellulase activity until the 36 hour time
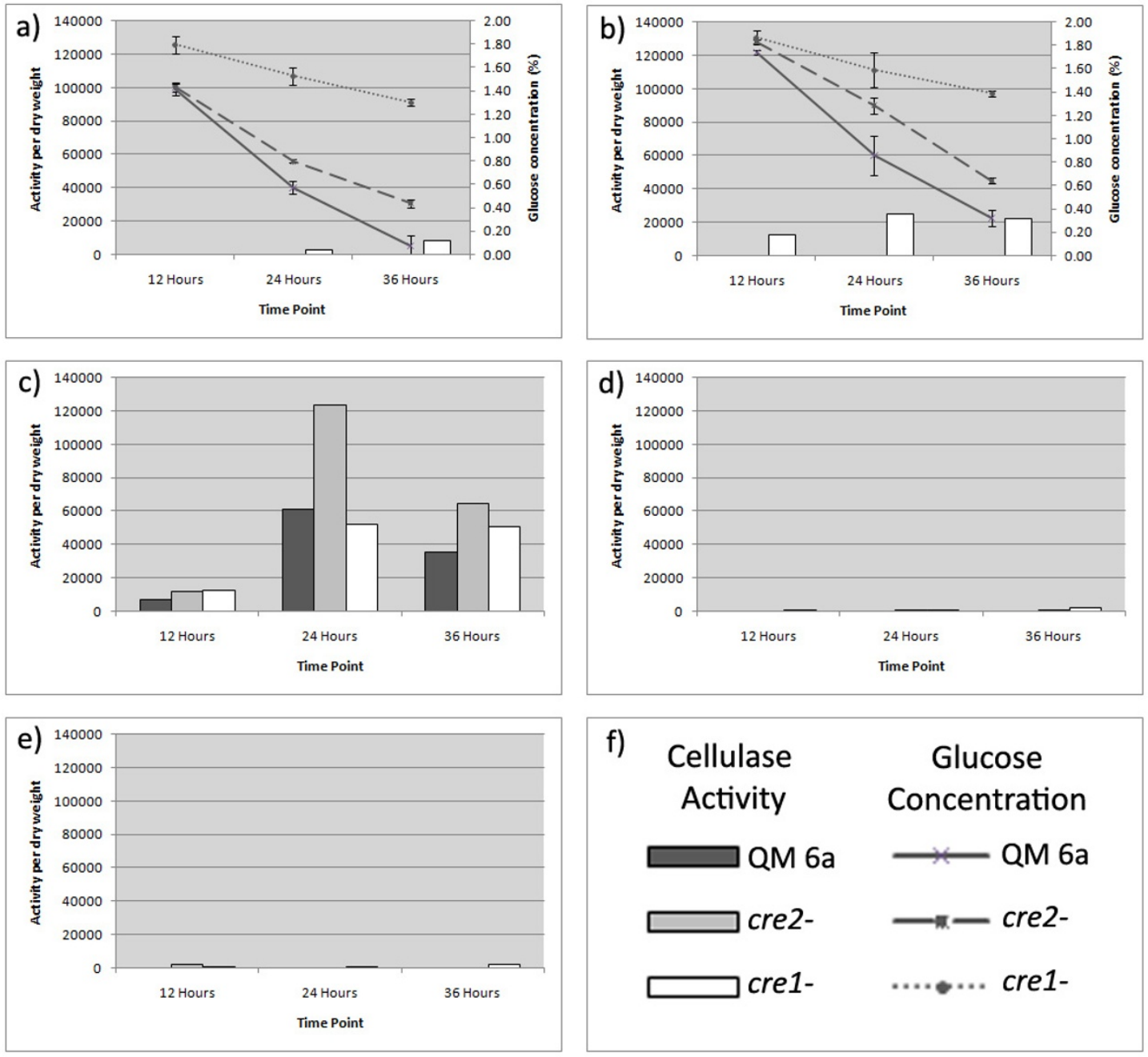

Figure 5 Total secreted Cellulase in Lactose induced cultures. Cellulase secretion per gram dry weight in T. reesei strains QM 6a, JKTR2-6 and crel deletion, 12, 24 and 36 hours post transfer. Mycelia for inoculation were harvested after growth for 24 hours in 1\% (w/v) glucose medium. Mycelia were washed with liquid carbon free medium and $200 \mathrm{mg}$ was added to $50 \mathrm{ml}$ culture medium in $250 \mathrm{ml}$ Erlenmeyer flasks. Cultures were grown at $30^{\circ} \mathrm{C}$, shaken at 200 RPM, and were harvested 12 hours, 24 hours and 36 hours post inoculation. Total secreted cellulase activity was measured using the EnzChek Cellulase Substrate expressed as fluorescence per mg. Dry weights are shown in Additional File 1 and levels of total cellulase activity are shown in Additional File 3. Glucose concentrations were determined by HPLC analysis. The carbon sources for each of the cultures are a) $2 \%$ glucose, b) $2 \%$ glucose and $2 \%$ lactose, c) $2 \%$ lactose, d) $2 \%$ sorbitol and e) $2 \%$ glycerol. The strain key is f). The data presented is based on biological triplicates that were assayed in duplicate. 
point in $2 \%$ lactose and glucose indicating very modest levels of derepression (Additional File 3). After 36 hours glucose concentrations in the cultures had dropped to $0.3 \%$ for QM 6a and $0.6 \%$ for JKTR2-6, but no activity could be detected in the QM 6a cultures. In medium containing lactose as an inducer, but lacking a repressing carbon source, cellulase activity per gram dry weight was higher in JKTR2-6 compared to QM 6a across all three time points, particularly at 24 hours (Figure 5c).

Total secreted xylanase activity was also measured in $2 \%$ glucose, $2 \%$ glucose $/ 2 \%$ lactose and $2 \%$ lactose cultures. As with the cellulase assays, JKTR2-6 showed elevated total activity by weight when compared to QM 6a on $2 \%$ lactose (Additional File 3).

\section{Microcrystalline cellulose as inducer}

Since elevated expression was observed with lactose as an inducer after 24 hours induction, we also tested this time point using Avicel and carboxy methyl cellulose as inducers. We grew $100 \mathrm{ml}$ cultures of QM 6a and JKTR2-6 for 24 hours in media containing $0.5 \%$ avicel, with or without $1 \%$ glucose. In both QM 6a and JKTR26 there was no detectable cellulase activity in any of the cultures containing glucose. In the cultures containing inducer without repressor, there was significantly more cellulase activity per gram dry-weight mycelium in JKTR2-6 compared to QM 6a (Figure 6). We also grew

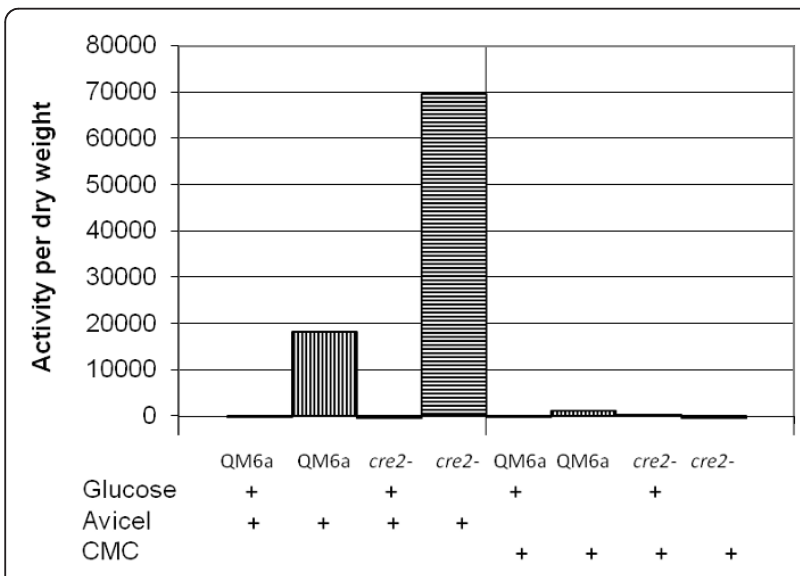

Figure 6 Total secreted cellulase in Avicel induced cultures. Cellulase secretion in T. reesei strains QM 6a and JKTR2-6 grown for 24 hours post transfer. Conidia were added to $100 \mathrm{ml}$ culture medium in $250 \mathrm{ml}$ Erlenmeyer flasks. Cultures were grown at $30^{\circ} \mathrm{C}$, shaken at 1500 RPM. Total secreted cellulase activity was measured using the EnzChek Cellulase Substrate expressed as fluorescence/mg dry weight. Dry weights are shown in Additional File 4. The carbon sources shown are $1 \%$ glucose plus $0.5 \%$ avicel, $0.1 \%$ fructose plus $0.5 \%$ avicel, $1 \%$ glucose plus $0.5 \%$ carboxymethyl cellulose, and $0.1 \%$ fructose plus $0.5 \%$ carboxymethyl cellulose. No cellulase was detected using this detection system in either strain or condition using CMC as inducer. The data presented is based on biological triplicates that were assayed in duplicate.
$100 \mathrm{ml}$ cultures of QM 6a and JKTR2-6 for 24 hours in media containing $0.5 \%$ carboxymethyl cellulose, with or without $1 \%$ glucose, but in these culture conditions, carboxymethyl cellulose did not induce any cellulase that was detectable using the ENZ-CHEK cellulase assay substrate as the detection system. Because carboxy methyl cellulase has been detected in these conditions previously, we assume that this is due to a limitation of the detection substrate.

\section{Discussion}

The $T$. reesei Cre 2 amino acid sequence is conserved with $A$. nidulans $\mathrm{CreB}$, particularly within the region encoding the ubiquitin C-terminal hydrolase domain, and complementation of the pleiotropic phenotype of the A. nidulans creB1937 mutant by the $T$. reesei cre 2 gene showed it is a functional orthologue of $c r e B$. The effects of $\mathrm{CreB}$ on carbon catabolite repression and the effects on the regulation of permeases are proposed to operate via separate mechanisms, and thus the complementation of the range of phenotypes indicates that both functions are conserved between the two orthologues.

While it is not uncommon for filamentous fungal genes expressed from their endogenous promoters to function in other fungal species, such as the amdS gene encoding acetamidase [29], CreB forms part of a large complex with CreC [10], and is likely to require multiple protein-protein interactions for its functions. Therefore the complementation of the creB1937 mutation by cre 2 is of importance as it demonstrates that the $\mathrm{CreB} / \mathrm{CreC}$ complex previously identified in $A$. nidulans, and also the targets of $\mathrm{CreB}$ deubiquination, are conserved between distant filamentous fungi despite relatively low conservation outside of the ubiquitin C-terminal hydrolase domain.

We made a cre 2 disruptant strain using molecular genetic techniques, and the phenotype of the disruptant is consistent with published phenotypes of $\mathrm{creB}$ mutations in $A$. nidulans. We used both Southern analysis and meiotic crossing to show that the cre 2 disruption was the result of a double cross over event at the cre 2 locus, and that the phenotype was due to the cre 2 disruption and not to a fortuitous alteration to the genome that occurred in the transformation. The discovery of a sexual cycle in $T$. reesei [27] allows for the first time traditional genetic analysis of industrial strains, allowing alternatives to molecular approaches. In this case, a meiotic cross was used to demonstrate that the selectable marker phenotype and the cre 2 disruption phenotype co-segregate, thus strengthening that the disruption phenotype was due to the cre 2 disruption and not to a fortuitous alteration to the genome.

Growth testing on both solid and liquid media revealed similar phenotypes between $T$. reesei and $A$. 
nidulans strains containing $\mathrm{cre} 2 / \mathrm{creB}$ mutations. These growth tests support the initial hypothesis that a strain with disrupted cre 2 will lack the extreme morphological impairment of cre 1 mutants, although the disruption of cre 2 does lead to somewhat impaired growth on glucose and lactose, evident in liquid culture.

In the absence of glucose, disruption of cre 2 leads to elevated cellulase activity whether sophorose, lactose or microcrystalline cellulose was used as an inducer, but not when a source of induction was absent, and this elevated cellulase activity in induced conditions is a robust phenotype of the cre 2 disruption. When glucose is also present with an inducer, disruption of cre2 leads to only a very slight relaxation of glucose repression compared to QM 6a, however this derepression is much less than that due to disruption of cre1.

A full description of the functional roles of $\mathrm{CreB}$ has yet to be determined, even in A. nidulans. The CreB protein has been shown to be a functional deubiquitinase in an $E$. coli assay (9), and its direct effect on permeases have been shown for the quinate permease (16; Kamlangdee and Kelly, unpublished), and effects on permeases are likely to account for the reduced growth on some sole carbon sources. In addition to these effects on growth in various carbon sources, the $A$. nidulans cre $B$ mutations also lead to carbon catabolite derepression of a number of enzyme activities in the presence of a source of repression, including alcohol dehydrogenase induction by ethanol which requires no permease. Thus the full range of pleiotropic phenotypes are unlikely to be accounted for solely by effects on permeases. The range of phenotypes due to lack of function mutations in A. nidulans (9), Aspergillus oryzae (Hunter and Kelly, unpublished) and $T$. reesei orthologues are broadly similar, and thus the functions are likely to be conserved across these organisms. The effects on the induction of activities of enzymes found to be elevated in mutations in $A$. nidulans and $A$. oryzae involve effects on transcription, and preliminary results indicate that this is likely also to be the case in $T$. reesei (Morris, Hunter and Kelly, unpublished). Thus it is likely that CreB plays a role in transcriptional regulation, as well as its effects on the stability of permeases.

Since the disruption of cre 2 increases the amount of cellulase activity without the severe morphological deficiencies seen with the cre 1 disruption, targeting creB orthologues for disruption in other industrially useful filamentous fungi, such as A. oryzae, Trichoderma harzianum or A. niger may also prove beneficial. While the disruption of cre2 has increased secreted cellulase activity, further improvements could potentially be made using JKTR2-6 as a foundation for further targeted genetic manipulation. Examples of potential manipulations include making a cre 1 cre 2 double null strain, and the over expression of the $T$. reesei orthologue of $\mathrm{creD}$ [30], shown in $A$. nidulans to increase derepression in a creB null background (R. Lockington, personal communication). Protease encoding genes have previously been targeted for disruption to improve heterologous protein expression in both $A$. oryzae [31,32] and A. niger [33]. T. reesei proteases could also be targeted in these strains to potentially improve extracellular cellulase activity.

\section{Conclusions}

We have identified and disrupted the $c r e 2$ gene in $T$. ree$s e i$, and shown that it is the functional homologue of the creB gene in $A$. nidulans. The disrupted strain shows a similar phenotype to the equivalent $A$. nidulans mutant. We have shown that the disruption of cre 2 increases the amount of cellulase activity in the presence of an inducer, without severe morphological affects. Therefore, we propose that targeting $c r e B$ orthologues for disruption in other industrially useful filamentous fungi, such as $A$. oryzae, T. harzianum or A. niger may also lead to elevated hydrolytic enzyme activity in these species, and we are presently investigating these possibilities.

\section{Methods}

\section{Strains}

The $A$. nidulans strains used were creB1937 (yA1 pabaA1; creB1937; riboB2) and wild-type (yA1 pabaA1; riboB2). The $T$. reesei strains used were QM 6a (wild type), CBS999.97 (containing MAT1-1, (27)), VTT-D 02877 (containing cre1::amdS, [17]) and JKTR2-6 (containing cre2::amdS, this study). Escherichia coli strain DH5a (supE44 AlacU169 (Ф80 lacZ $\Delta M 15)$ hsdR17 recA1 endA1 gyrA96 thi-1 relA1) was used to propagate plasmids.

\section{Media}

Solid and $5 \mathrm{ml}$ liquid fungal media were based on that described by Cove [34] or potato dextrose agar (PDA) where stated, while $50 \mathrm{ml}$ and $100 \mathrm{ml}$ liquid medium was as described by Seiboth et al. [35]. A. nidulans and T. reesei were grown at $37^{\circ} \mathrm{C}$ and $30^{\circ} \mathrm{C}$ respectively. Nitrogen sources were added to a final concentration of $10 \mathrm{mM}$ and carbon sources at $1 \%$ (wt/vol), unless otherwise stated. Riboflavine and para amino benzoic acid were added to media at final concentrations of $2.5 \mu \mathrm{g}$ per $\mathrm{ml}$ and $0.5 \mu \mathrm{g}$ per $\mathrm{ml}$, respectively, when required. All $T$. reesei growth tests were initially conducted on detergent free solid media but for photography, TritonX-100 was added to $T$. reesei media at $0.01 \%$ (vol/ vol) to reduce colony diameter.

\section{T. reesei Sexual Cross}

The $T$. reesei sexual cross was performed as described in Seidl et al. [27] between JKTR2-6 (cre2::amdS) and CBS999.97 (MAT1-1) on PDA at $25^{\circ} \mathrm{C}$ with 16 hours of 
light in a 24 hour period. Fruiting bodies formed approximately 16 days post inoculation and spores were collected from the lid of the petri dish on day 22 .

\section{Transformation}

A. nidulans was transformed based on the method described by Tilburn et al. [36], and transformants were selected for riboflavine independence conferred by the ribo $B^{+}$gene in pPL3 [37]. T. reesei was transformed using linearised plasmid based on the method described by Pentillä et. al [38]. Transformants were selected on medium containing $10 \mathrm{mM}$ acetamide as the sole nitrogen source using the amdI9 variant of the amdS gene from $A$. nidulans. This amdI9 variant leads to higher expression of acetamidase, and thus easier selection of single copy transformants. Bacteria were transformed as described in Sambrook and Russell [39].

\section{Molecular methods}

Molecular methods were as described by Sambrook and Russell [39]. Bacterial plasmids were purified using the Wizard Plus SV Minipreps DNA Purification System (Promega, USA). Fungal DNA was extracted using the DNeasy Plant Mini Kit DNA purification system (Qiagen, USA). All PCRs were performed using the high fidelity polymerase, Phusion (Genesearch, Australia). Southern analysis was performed using DIG Highprime Labelling and Detection Kit (Roche, Australia).

\section{Generation of cre2 Constructs}

A 4505 nucleotide region encompassing the Cre 2 coding region, including approximately $500 \mathrm{bp}$ upstream of the putative start codon and approximately 200 bp downstream of the putative stop codon, was amplified using primers CBUP1 (5'CCCATTGCTGTCTCGCTATT3') and CBDOWN2 (5'AAGGCAAGATGTGTCGGAAC3'). The amplicon was cloned into pBluescript generating pTRcre2 for use in heterologous complementation analysis. The disruption construct, pTRcre2 $\Delta$ amdS, was generated through ligation of the $A$. nidulans amdS encoding region, containing the amdI9 promoter mutation, into a MscI restriction site. This restriction site occurs prior to the codon for His426, which is within the 300 amino acid ubiquitin hydrolase domain and prior to His428 and Asp437 shown to be active sites [26].

\section{Analytical Methods}

Mycelia from fungal strains QM 6a, VTT-D 02877, the cre1 disruption, and JKTR2-6, the cre2 disruption, were cultured either in small $5 \mathrm{ml}$ cultures in A. nidulans medium described by Cove [34], on in larger $50 \mathrm{ml}$ or $100 \mathrm{ml}$ cultures in $T$. reesei medium described by Seiboth et al. [35].
Total cellulase or xylanase enzyme activity was assayed either using the ENZ-CHEK cellulase assay substrate or the ENZ-CHEK xylanase assay kit (Invitrogen, USA) following the manufacturer's instructions, using a Molecular Devices SpectraMax Plus384 Absorbance Microplate Reader. These assays determine the relative cellulase or xylanase activity between strains within a single experiment, by the measurement of fluorescence at $360 / 460 \mathrm{~nm}$ produced by cellulase or xylanase activity on the substrates. The manufacturer's standards indicate that a fluorescence of 40000 corresponds to approximately $6 \mathrm{mU} / \mathrm{mL}$ of cellulase activity for the cellulase substrate, while a fluorescence of 1000 corresponds to approximately 450 $\mathrm{mU} / \mathrm{mL}$ of xylanase activity using the xylanase kit.

The biomass from each culture was harvested, dried at $65^{\circ} \mathrm{C}$ and weighed.

A Rezex ROA-Organic analysis column $(300 \times 7.8$ $\mathrm{mM}$, Phenomenez, Australia) and a refractive index detector (Model 350, Varian, Australia) were used to analyse glucose concentrations. The mobile phase was ultra pure water at a flow rate of $0.6 \mathrm{ml} / \mathrm{min}$ and the column was maintained at $35^{\circ} \mathrm{C}$.

\section{Additional material}

Additional file 1: Total Dry Biomass in Various Carbon Sources

Biomass was harvested from strains grown on carbon sources as shown for 12,24 and 36 hours at $30^{\circ} \mathrm{C}$. The strains are QM 6a, JKTR2-6, and the cre1 deletion strain. Dry weight is shown in $\mathrm{mg}$ per $\mathrm{ml}$.

Additional file 2: Total secreted cellulase activity of three $T$. reesei strains. Cellulase secretion of T. reesei strains QM 6a (dark grey shading), JKTR2-6 (light grey shading) and crel deletion (white shading) measured using the EnzChek Cellulase Substrate. Mycelia for inoculation were harvested after growth for 28 hours in $1 \%(\mathrm{w} / \mathrm{v})$ glucose medium. Mycelia were washed with liquid carbon free medium and $50 \mathrm{mg}$ was added to $5 \mathrm{ml}$ culture medium in $10 \mathrm{ml}$ culture bottles. Cultures were grown at $30^{\circ} \mathrm{C}$, shaken at 200 RPM, and were harvested 6 hours, 12 hours and 18 hours post inoculation. Time indicated as hours post transfer. Error bars indicate standard deviations of duplicate cultures each analysed in duplicate. (a) Repressing conditions, growth in medium containing 1\% glucose and $1 \mathrm{mM}$ sophorose. (b) Derepressing conditions, growth in medium containing $1 \%$ sorbitol and $1 \mathrm{mM}$ sophorose. When diluted, the measured cellulase activity of these samples decreased proportionally, showing the detection limit of the assay had not been exceeded.

Additional file 3: Secreted Cellulase and Xylanase Activities. Cellulase (a) or Xylanase (b) secretion in T. reesei strains QM 6a, JKTR2-6, and the crel disruption strain measured using the EnzChek Cellulase Substrate (a) or the EnzChek Xylanase Assay Kit (b) uncorrected for dry weight. Time indicated as hours post transfer, grown at $30^{\circ} \mathrm{C}$. Error margins indicated standard deviations determined from the result of assay duplicates and biological triplicates.

Additional file 4: Dry weights for Avicel and Carboxymethyl Cellulose cultures. Data for Figure 6. Conidia were added to $100 \mathrm{ml}$ culture medium in $250 \mathrm{ml}$ Erlenmeyer flasks. Cultures were grown at $30^{\circ}$ C, shaken at 1500 RPM, for 24 hours, in triplicate. The carbon sources are $1 \%$ glucose plus $0.5 \%$ avicel, $0.1 \%$ fructose plus $0.5 \%$ avicel, $1 \%$ glucose plus $0.5 \%$ carboxymethyl cellulose, $0.1 \%$ fructose plus $0.5 \%$ carboxymethyl cellulose. 


\section{Acknowledgements}

JD was supported by a scholarship from the University of Adelaide. We thank Dr Robin Lockington for fruitful discussions regarding initial project design; Mr Tong Tong Wang for technical assistance; Prof. M. Pentillä for providing T. reesei strains QM 6a and VIT-D 02877; Dr. M. Schmoll for providing T. reesei strain CBS999.97; Mr Adrian Hunter for assistance with HPLC; and Mr Adrian Hunter and Ms Vivian Georgakopoulos for critical reading of the manuscript.

\section{Authors' contributions}

JD carried out project design, research work and drafting of the manuscript. JK was responsible for supervision, initial project conception and editing of the manuscript. All authors read and approved the final manuscript.

Received: 20 June 2011 Accepted: 9 November 2011

Published: 9 November 2011

\section{References}

1. Kubicek CP, Mikus M, Schuster A, Schmoll M, Seiboth B: Metabolic engineering strategies for improvement of cellulase production by Hypocrea jecorina. Biotechnol Biofuels 2009, 2:19.

2. Stricker AR, Mach RL, de Graaff LH: Regulation of transcription of cellulasesand hemicellulases-encoding genes in Aspergillus niger and Hypocrea jecorina (Trichoderma reesei). Appl Microbiol Biotechnol 2008, 78:211-20.

3. Ilmen M, Thrane C, Penttilä M: The glucose repressor gene cre1 of Trichoderma: isolation and expression of a full-length and a truncated mutant form. Molec Gen Genet 1996, 251:451-460.

4. Seidl V, Gamauf C, Druzhinina IS, Seiboth B, Hartl L, Kubicek CP: The Hypocrea jecorina (Trichoderma reesei) hypercellulolytic mutant RUT C30 lacks a $85 \mathrm{~kb}$ (29 gene-encoding) region of the wild-type genome. BMC Genomics 2008, 9:327.

5. Geysens S, Pakula T, Uusitalo J, Dewerte I, Penttilä M, Contreras R: Cloning and characterization of the glucosidase II alpha subunit gene of Trichoderma reesei: a frameshift mutation results in the aberrant glycosylation profile of the hypercellulolytic strain Rut-C30. Appl Environ Microbiol 2005, 71:2910-24.

6. Marika Vitikainen M, Arvas M, Pakula T, Oja M, Penttilä M, Saloheimo M: Array comparative genomic hybridization analysis of Trichoderma reesei strains with enhanced cellulase production properties. BMC Genomics 2010, 11:441.

7. Dowzer CEA, Kelly JM: Analysis of the creA gene, a regulator of carbon catabolite repression in Aspergillus nidulans. Molec Cell Biol 1991, 11:5701-5709.

8. Dowzer CEA, Kelly JM: Cloning of creA from Aspergillus nidulans: a gene involved in carbon catabolite repression. Current Genet 1989, 15:457-459.

9. Lockington RA, Kelly JM: Carbon catabolite repression in Aspergillus nidulans involves deubiquitination. Molec Microbiol 2001, 40:1311-21.

10. Lockington RA, Kelly JM: The WD40-repeat protein CreC interacts with and stabilizes the deubiquitinating enzyme CreB in vivo in Aspergillus nidulans. Molec Microbiol 2002, 43:1173-82.

11. Drysdale MR, Kolze SE, Kelly JM: The Aspergillus niger carbon catabolite repressor encoding gene, creA. Gene 1993, 130:241-245.

12. Takashima S, Nakamura A, Hidaka M, Masaki H, Uozumi T: Isolation of the creA gene from the cellulolytic fungus Humicola grisea and analysis of CreA binding sites upstream from the cellulase genes. Biosci Biotechnol Biochem 1998, 62:2364-70.

13. Tonukari NJ, Scott-Craig JS, Walton JD: Isolation of the carbon catabolite repressor (CREA) gene from the plant-pathogenic fungus Cochliobolus carbonum. DNA Sea 2003, 14:103-7.

14. Tudzynski B, Liu S, Kelly JM: Carbon catabolite repression in plant pathogenic fungi: isolation and characterization of the Gibberella fujikuroi and Botrytis cinerea creA genes. FEMS Microbiol Letts 2000, 184:9-15.

15. Hynes MJ, Kelly JM: Pleiotropic mutants of Aspergillus nidulans altered in carbon metabolism. Molec Gen Genet 1977, 150:193-204.

16. Kamlangdee N: Identifying target proteins of the CreB deubiquitination enzyme in the fungus Aspergillus nidulans. Phd Thesis University of Adelaide, Adelaide; 2007.

17. Nakari-Setala T, Paloheimo M, Kallio J, Vehmaanpera J, Penttilä M, Saloheimo M: Genetic modification of carbon catabolite repression in Trichoderma reesei for improved protein production. Appl Environ Microbiol 2009, 75:4853-4860.
18. Aro N, Ilmen M, Saloheimo A, Penttilä M: ACEl of Trichoderma reesei is a repressor of cellulase and xylanase expression. Appl Environ Microbiol 2003, 69:56-65.

19. Saloheimo A, Aro N, Imen M, Penttilä M: Isolation of the ace1 gene encoding a Cys(2)-His(2) transcription factor involved in regulation of activity of the cellulase promoter cbh1 of Trichoderma reesei. J Biol Chem 2000, 275:5817-25.

20. Aro N, Saloheimo A, Imen M, Penttilä M: ACEII, a Novel Transcriptional Activator Involved in Regulation of Cellulase and Xylanase Genes of Trichoderma reesei. J Biol Chem 2001, 276:24309-24314.

21. Rauscher R, Wurleitner E, Wacenovsky C, Aro N, Stricker AR, Zeilinger S, Kubicek CP, Penttilä M, Mach RL: Transcriptional regulation of xyn1, encoding xylanase I, in Hypocrea jecorina. Euk Cell 2006, 5:447-56.

22. Stricker AR, Grosstessner-Hain K, Wurleitner E, Mach RL: Xyr1 (xylanase regulator 1 ) regulates both the hydrolytic enzyme system and D-xylose metabolism in Hypocrea jecorina. Euk Cell 2006, 5:2128-37.

23. Mach-Aigner AR, Pucher ME, Steiger MG, Bauer GE, Preis SJ, Mach RL: Transcriptional regulation of $x y r 1$, encoding the main regulator of the xylanolytic and cellulolytic enzyme system in Hypocrea jecorina. Appl Environ Microbiol 2008, 74:6554-62.

24. Shida Y, Furukawa T, Ogasawara W, Kato M, Kobayashi T, Okada H, Morikawa Y: Functional analysis of the eg/3 upstream region in filamentous fungus Trichoderma reesei. Appl Microbiol Biotechnol 2008, 78:515-24.

25. Stricker AR, Steiger MG, Mach RL: Xyr1 receives the lactose induction signal and regulates lactose metabolism in Hypocrea jecorina. FEBS Letts 2007, 581:3915-20.

26. Hu M, Li P, Li M, Li W, Yao T, Wu JW, Gu WR, Cohen E, Shi Y: Crystal structure of a UBP-family deubiquitinating enzyme in isolation and in complex with ubiquitin aldehyde. Cell 2002, 111:1041-54.

27. Seidl V, Seibel C, Kubicek CP, Schmoll M: Sexual development in the industrial workhorse Trichoderma reesei. Proc Natl Acad Sci USA 2009 106:13909-14.

28. Kubicek CP, Messner R, Gruber F, Kubicek-Pranz EM: Triggering of cellulose biosynthesis in Trichoderma reesei: involvement of a constitutive, sophorose inducible, glucose inhibited B-diglucoside permease. J Biol Chem 1993, 268:19364-19368.

29. Kelly JM, Hynes MJ: Transformation of Aspergillus niger by the amdS gene of Aspergillus nidulans. EMBO J 1985, 4:475-9.

30. Boase NA, Kelly JM: A role for creD, a carbon catabolite repression gene from Aspergillus nidulans, in ubiquitination. Molec Microbiol 2004, 53:929-940.

31. Jin FJ, Watanabe T, Juvvadi PR, Maruyama J, Arioka M, Kitamoto K: Double disruption of the proteinase genes, tppA and pepE, increases the production level of human lysozyme by Aspergillus oryzae. Appl Microbiol Biotechnol 2007, 76:1059-68.

32. Yoon J, Kimura S, Maruyama J, Kitamoto K: Construction of quintuple protease gene disruptant for heterologous protein production in Aspergillus oryzae. Appl Microbiol Biotechnol 2009, 82:691-701.

33. van den Hombergh JP, Sollewijn Gelpke MD, van de Vondervoort PJ, Buxton FP, Visser J: Disruption of three acid proteases in Aspergillus niger-effects on protease spectrum, intracellular proteolysis, and degradation of target proteins. Eur J Biochem 1997, 247:605-13.

34. Cove DJ: The induction and repression of nitrate reductase in the fungus Aspergillus nidulans. Biochim Biophys Acta 1966, 113:51-6.

35. Seiboth B, Hakola S, Mach RL, Suominen PL, Kubicek CP: Role of four major cellulases in triggering of cellulase gene expression by cellulose in Trichoderma reesei. J Bacteriol 1997, 179:5318-5320.

36. Tilburn J, Scazzocchio C, Taylor GG, Zabicky-Zissman JH, Lockington RA, Davies RW: Transformation by integration in Aspergillus nidulans. Gene 1983, 26:205-21.

37. Oakley CE, Weil CF, Kretz PL, Oakley BR: Cloning of the riboB locus of Aspergillus nidulans. Gene 1987, 53:293-8.

38. Penttilä $M$, Nevalainen $H$, Ratto $M$, Salminen $E$, Knowles J: A versatile transformation system for the cellulolytic filamentous fungus Trichoderma reesei. Gene 1987, 61:155-64.

39. Sambrook J, Russell DW: Molecular Cloning: A Laboratory Manual. Cold Spring Harbor Laboratory Press, Cold Spring Harbor, New York; Third 2001.

doi:10.1186/1472-6750-11-103

Cite this article as: Denton and Kelly: Disruption of Trichoderma reesei cre2, encoding an ubiquitin C-terminal hydrolase, results in increased cellulase activity. BMC Biotechnology 2011 11:103. 